\title{
Impact of Physician-Pharmacist Collaboration on Diabetes Outcomes and Health Care Use
}

\author{
Melissa C. Norton, MS, PharmD, Meghan E. Haftman, PharmD, BCPS, and \\ Lyndsey N. Buzzard, PharmD, BCACP
}

Objective: To evaluate the impact of physician-pharmacist collaboration for disease-state management on diabetes outcomes in primary care by comparing outcomes between physician-managed care and pharmacist collaborative care.

Methods: A retrospective, observational cohort study was conducted at Ascension Medical Group Via Christi, P.A. from January 1, 2016 to June 30, 2018. Health outcomes were analyzed in 385 patients with diabetes mellitus collaboratively managed by a physician and pharmacist (collaborative care group). Similar patients managed by physician only (usual care group) were matched to the collaborative care group using nearest neighbor matching. The primary outcome compared glycosylated hemoglobin (HbA1c) change between collaborative care and usual care groups at 12 months.

Results: The mean change in HbA1c decreased by $1.75 \%$ in the collaborative care group and $0.16 \%$ in the usual care group $(P<.0001)$. The usual care group had a larger number of patients with HbA1c less than $8 \%$ at follow-up $(P=.0049)$. Additional outcomes included decrease in total cholesterol $(P=.0023)$, triglycerides $(P=.0016)$, and an increase in PPSV23 pneumococcal vaccinations $(P=.0255)$ in the collaborative care group. The usual care group had an increase in PCV13 pneumococcal vaccinations $(P=.0075)$. Both emergency department visits $(P=.0162)$ and hospitalizations $(P=.0225)$ decreased significantly in the collaborative care group, estimating total savings of $\$ 633,015$.

Conclusions: The collaboration of pharmacists and physicians in the primary care setting is associated with improved diabetes outcomes and substantially reduces costs related to decreased health care use. (J Am Board Fam Med 2020;33:745-753.)

Keywords: Cohort Studies, Cost Savings, Diabetes Mellitus, Glycosylated Hemoglobin, Managed Care Programs, Pharmacists, Physicians, Practice-Based Research, Primary Health Care, Retrospective Studies

\section{Introduction}

The role of pharmacists in the outpatient, nonacute care setting has expanded from the traditional dispensing roles in community pharmacies to a clinical role with direct patient care responsibilities within various ambulatory care settings, including primary care clinics. ${ }^{1,2}$ In many collaborative care models within a primary care group practice, clinical pharmacists are embedded in the practice and are

This article was externally peer reviewed.

Submitted 28 January 2020; revised 25 May 2020; accepted 26 May 2020.

From the Ascension Medical Group Via Christi, P.A., Wichita, KS

Funding: None.

Conflicts of interest: None.

Corresponding author: Melissa C. Norton, MS, PharmD, 1100 N St Francis St, Suite 220, Wichita, KS 67214 (E-mail: melissa.norton@ascension.org). acknowledged as a member of the primary care team. ${ }^{3}$ Pharmacists within the primary care setting practice in collaboration with physicians and provide clinical pharmacy services that may include counseling and medication education, comprehensive medication management, chronic care management, and population health management for a variety of disease states. ${ }^{2-13}$ As of 2016, 48 states in the United States allow pharmacists and prescribers to enter into a collaborative practice agreement, although specific restrictions and regulations vary by state. ${ }^{14}$ Collaborative practice agreements provide pharmacists the ability to manage chronic disease states in collaboration with physicians to improve patient outcomes and patient satisfaction. ${ }^{5,14-16}$

The involvement of clinical pharmacists in direct patient care has been shown to improve clinical outcomes in many chronic disease states, including but 
not limited to diabetes, hypertension, and dyslipidemia. ${ }^{2,6-9,12,13,16-20}$ Data also supports that clinical pharmacy services improve provider work-life ${ }^{21}$ and reduce health care costs..$^{12,13,16,17,22,23}$ Matzke et $\mathrm{al}^{17}$ found that the inclusion of clinical pharmacists in a collaborative care model demonstrated significant improvements in glycosylated hemoglobin, blood pressure, low-density lipoprotein (LDL) cholesterol, and total cholesterol in the collaborative care group compared with the usual care group. The study also revealed that hospitalizations in the collaborative care group were reduced by $23.4 \%$, yielding an estimated cost savings of $\$ 2619$ per patient and a return on investment of 504\%. ${ }^{17}$

In an effort to decrease health care costs, enhance patient experiences, and improve outcomes, a teambased approach to direct patient care has been acknowledged as an important component to improve health care quality, with pharmacists recognized as a vital member of the health care team. ${ }^{4,15}$ With the high prevalence of diabetes, along with the complex medication management and the association of high health care costs, there is a substantial amount of literature to support the involvement of clinical pharmacists in diabetes management to improve and meet clinical goals. ${ }^{2,6,9,16-19,23-25}$ Despite the support of published literature showing the effect of clinical pharmacists on improvement of patient outcomes, very few studies compare collaborative pharmacist management to that of physician-only care in a primary care setting. In addition, data for cost evaluation of clinical pharmacy services in a primary care clinic is limited.

The implementation of clinical pharmacy services in primary care at Ascension Medical Group Via Christi, P.A. began in 2012, yet formal evaluation of the impact of those services has yet to be performed. The purpose of this study was to evaluate the impact of physician-pharmacist collaborative disease state management on diabetes outcomes and health care use compared with usual care patients, which consisted of physician-managed care for diabetes.

\section{Methods}

\section{Setting}

This study was conducted at Ascension Medical Group Via Christi, P.A. which is the network of primary and specialty care clinics within Ascension Via Christi Health in Wichita, Kansas, and represents the largest multi-specialty group practice in
Kansas. Within this clinic network, there are 9 clinic locations specializing in family medicine and internal medicine, in which at the time of this study, 6 of the locations had clinical pharmacy services with embedded clinical pharmacists working in collaboration with physicians. Patients within the clinic are identified for referral to the clinical pharmacists by clinic staff and providers to meet with the patients one on one at separate visits for comanagement of medications and disease states within their scope of practice through a broad collaborative practice agreement that is not restricted to specific disease states or medication classes. Kansas laws allow for pharmacists to have collaborative practice agreements with individual physicians. The scope of practice for the pharmacist is defined by federal law and state laws and further described within the collaborative practice agreement based on a mutual agreement with the physician(s). The collaborative practice agreements allow the clinical pharmacists to initiate, adjust, and stop medications, as well as order laboratory monitoring, but do not allow pharmacists to be independent prescribing practitioners. In addition to clinical services, the pharmacists serve as resources for the clinics for drug information, clinical guideline recommendations, and provider and staff education.

\section{Study Design and Patient Population}

This study was a retrospective, observational cohort of patients with diabetes designed to compare patients managed by a primary care physician alone versus patients collaboratively managed by a physician-pharmacist team. Patients in the primary care clinic with a diagnosis of diabetes mellitus who had utilized clinical pharmacy services (collaborative care group) were included and matched to a cohort of similar control patients who experienced physician care without the collaboration of a pharmacist (usual care group) for comparison.

The collaborative care group consisted of patients who were established with a physician within the clinic and newly referred to a clinical pharmacist for collaborative diabetes management in 6 primary care clinic locations with an embedded clinical pharmacist. Patients were included who had a new patient visit and a minimum of 1 follow-up visit for diabetes within 6 months with a clinical pharmacist for collaborative diabetes management between the dates of January 1, 2016 and June 30, 2018. The new patient visit designated the start of 
physician-pharmacist collaborative care. Usual care patients were identified from 3 primary care clinics without an embedded clinical pharmacist that had 2 or more clinic encounters for diabetes within 6 months with a clinic provider during the same time frame.

Patients were eligible for study inclusion in the collaborative care group if they met the following inclusion criteria: 1) 18 years of age or older; 2) clinic encounter for a new patient visit with a clinical pharmacist; 3 ) 1 or more follow-up visit(s) with a clinical pharmacist within 6 months of new patient visit; and 4) documented ICD-10 code for diabetes during encounters. Patients were eligible for study inclusion in the usual care group if they met the following inclusion criteria: 1) 18 years of age or older; 2) 2 or more primary care provider clinic encounters within 6 months; and 3) documented ICD-10 code for diabetes during provider encounters. Patients in both groups were excluded if 1) the patient did not have a baseline and follow-up visit within 6 months; or 2) the patient did not have a documented baseline and follow-up glycosylated hemoglobin (HbA1c) value. This study was approved by the local institutional review board and informed consent was not required.

\section{Data Collection}

Date of entry into the study was defined as the date of new patient visit with the clinical pharmacist for the collaborative care group and date of clinic encounter with a provider for the usual care group. Clinical and therapeutic baseline data were retrospectively extracted from the electronic health record 90 days before and up to 21 days after the date of entry into the study with the closest measurements to the date of entry into the study defined as baseline measurements. Follow-up measurements were defined as the latest reported measurements within 12 months of date of entry into the study.

\section{Study Outcomes}

The primary outcome was the comparison of the mean absolute change in $\mathrm{HbA1c}$ from baseline between collaborative care and usual care groups. The secondary outcomes addressed additional diabetes metrics and included a comparison of the following between the collaborative care and usual care groups: percentage of patients with HbA1c less than $8 \%$ at follow-up; mean absolute change in systolic and diastolic blood pressure; mean absolute change in total cholesterol, LDL cholesterol, and triglycerides; and mean absolute change in pneumococcal vaccination, aspirin, and statin rates. An additional secondary outcome compared the change in number of all-cause emergency department (ED) visits and hospitalizations for 12 months before and 12 months post-study entry between collaborative care and usual care groups.

\section{Matched Cohort}

To control for patient characteristics between groups and minimize bias, patients from the collaborative care group $(n=385)$ were matched to patients from the usual care group $(\mathrm{n}=1290)$ after application of inclusion and exclusion criteria. Propensity score matching was used to match patients in the usual care group on a one-to-one basis to patients in the collaborative care group using nearest-neighbor matching. Matching variables consisted of change in $\mathrm{HbA1c}$ before study entry, age, gender, insulin status, and insurance type. Matching criteria was chosen based on common reasons for referral to a clinical pharmacist, including an increase in $\mathrm{HbAlc}$, need for insulin initiation or adjustments, and medication affordability issues. The types of insurance were organized into 3 categories: government (defined as Medicare, Medicaid, or other government programs); private or commercial; and self-pay or charity. Change in HbAlc was defined as the absolute change in $\mathrm{HbAlc}$ from latest recorded value within 12 months prior to the baseline value. If data was unavailable to calculate absolute change in HbA1c before baseline value, the change were recorded as $0 \%$, as not all study participants had $\mathrm{HbA1c}$ values available in the electronic health record 1 year before study entry. The utilization of propensity score calculations based on 5 patient variables and nearest-neighor matching minimized the effect that missing HbA1c values before study entry had on match quality.

\section{Statistical Analysis}

Demographic, clinical, and health care utilization characteristics were summarized for the study population at baseline using descriptive statistics and are presented in Table 1. All patient characteristics were evaluated using chi-square or independent $t$-test for categorical and continuous variables, respectively, to determine whether differences existed between groups. Continuous variables were described as means and $95 \%$ CIs and were analyzed using independent $t$ - 


\begin{tabular}{|c|c|c|c|}
\hline & Usual Care & Collaborative Care & $P$ value \\
\hline Age, $y$, mean $\pm \mathrm{SD}$ & $60.0 \pm 13.5$ & $60.8 \pm 13.2$ & .394 \\
\hline Male, No. (\%) & $190(49.4)$ & $205(53.3)$ & .279 \\
\hline Race, No. (\%) & & & .070 \\
\hline White & $319(82.9)$ & $338(87.8)$ & \\
\hline Black & $48(12.5)$ & $39(10.1)$ & \\
\hline Other & $18(4.7)$ & $8(2.1)$ & \\
\hline $\mathrm{BMI}, \mathrm{kg} / \mathrm{m}^{2}$, mean $\pm \mathrm{SD}$ & $34.8 \pm 8.1$ & $35.4 \pm 8.8$ & .601 \\
\hline eGFR, $\mathrm{mL} / \mathrm{min}(\%)$ & & & .029 \\
\hline$\geq 60$ & $227(69.4)$ & $243(69.0)$ & \\
\hline 45 to $<60$ & $63(19.3)$ & $52(14.8)$ & \\
\hline 30 to $<45$ & $29(8.9)$ & $53(15.1)$ & \\
\hline$<30$ & $8(2.4)$ & $4(1.1)$ & \\
\hline Diabetes diagnosis, No. (\%) & & & .362 \\
\hline Type 1 & $7(1.8)$ & $4(1.0)$ & \\
\hline Type 2 & $378(98.2)$ & $381(99.0)$ & \\
\hline $\mathrm{HbAlc} \%$, mean $\pm \mathrm{SD}$ & $7.57 \pm 1.62$ & $9.43 \pm 2.11$ & $<.001$ \\
\hline History of hypertension, No. (\%) & $316(82.1)$ & $295(76.6)$ & .062 \\
\hline History of hyperlipidemia, No. (\%) & $312(81.0)$ & $299(77.7)$ & .247 \\
\hline Health insurance & & & .641 \\
\hline Government & $190(49.4)$ & $202(52.5)$ & \\
\hline Commercial & $186(48.3)$ & $173(44.9)$ & \\
\hline Self-pay/charity & $9(2.3)$ & $10(2.6)$ & \\
\hline $\mathrm{ED}$ visits, mean $\pm \mathrm{SD}$ & $0.2 \pm 0.6$ & $0.3 \pm 0.9$ & .001 \\
\hline Hospital visits, mean $\pm \mathrm{SD}$ & $0.3 \pm 0.9$ & $0.6 \pm 1.3$ & .002 \\
\hline
\end{tabular}

BMI, body mass index; eGFR, estimated glomerular filtration rate; ED, emergency department; HbA1c, glycosylated hemoglobin; $\mathrm{SD}$, standard deviation.

test, evaluating the primary outcome of comparison of absolute change in $\mathrm{HbA1c}$ between groups and additional secondary outcomes. Secondary outcomes collected as categorical data were described as frequencies and analyzed using the chi-square test. The estimated sample size of 99 patients per group was calculated to achieve a power of $90 \%$ to detect an absolute mean between group difference in change in HbA1c of $1 \%$, assuming a 2 -sided test and a significance level $(\alpha)$ of 0.05 . To increase available data for secondary objectives, all eligible collaborative care participants were included in the analysis.

\section{Results}

A total of 3221 patients were screened for study inclusion with 828 identified in the collaborative care group and 2393 identified in the usual care group. After application of inclusion and exclusion criteria, 385 patients were included in the collaborative care group and 1290 were eligible for inclusion in the usual care group. The majority of patients excluded did not have a follow-up visit within 6 months or HbAlc within the allotted 12-month follow-up period. Usual care patients were matched to the 385 patients in the collaborative care group, with 905 usual care patients remaining unmatched, resulting in a total of 770 included patients.

Baseline characteristics were similar between groups with the exception of differences in estimated glomerular filtration rate between groups, and baseline HbA1c values as well as ED and hospital visits, which were significantly higher in the collaborative care group. The average age of the patients in each group was 60 years with comparable numbers of male and female patients in each group. The majority of the patients in each group were white and covered under a governmentadministered insurance. Complete baseline characteristics are described in Table 1.

The primary objective of change in HbAlc from baseline to follow-up between groups is represented in Table 2. The average baseline HbAlc for the usual care group was $7.57 \% \pm 1.62$ and decreased 


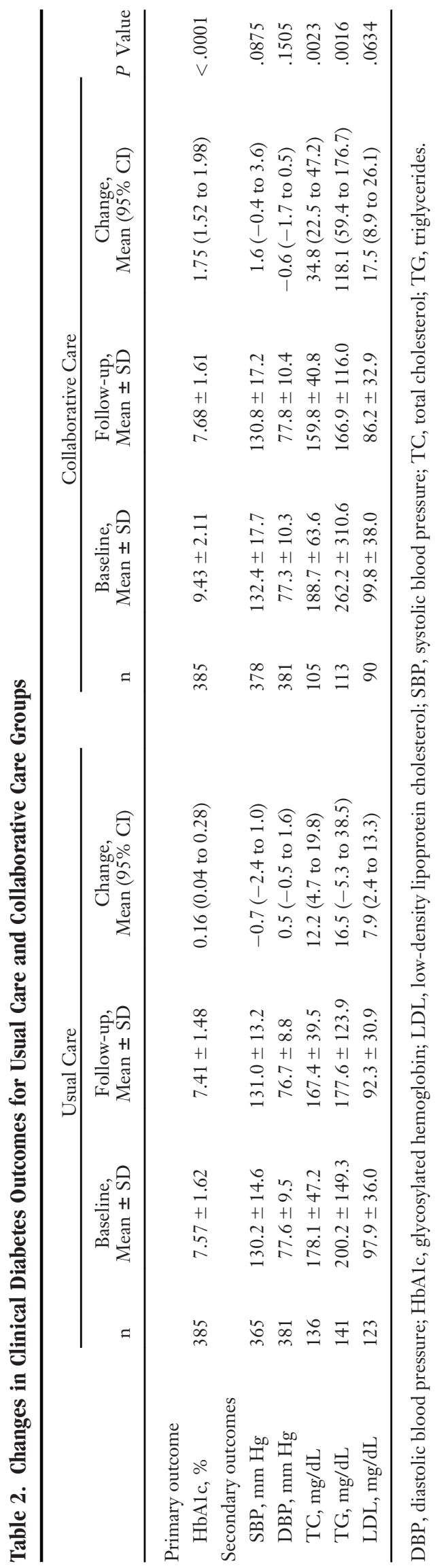

to $7.41 \% \pm 1.48$ at follow-up, an absolute mean change of $0.16 \%$ (0.04 to 0.28 ). The average $\mathrm{HbA1c}$ for the collaborative care group at baseline was $9.43 \% \pm 2.11$ and decreased to $7.68 \% \pm 1.61$ at follow-up, an absolute mean change of $1.75 \%$ (1.52 to 1.98), demonstrating a statistically significant difference in change in $\mathrm{HbA1}$ c between groups $(P<.0001)$. Additional clinical diabetes outcomes are represented in Table 2. Total cholesterol and triglycerides also showed a significant change in favor of collaborative care with a pharmacist. Total cholesterol and triglycerides decreased by $12.2 \mathrm{mg} /$ $\mathrm{dL}$ (4.7 to 19.8 ) and $16.5 \mathrm{mg} / \mathrm{dL}$ ( -5.3 to 38.5 ), respectively, in the usual care group compared with a decrease of $34.8 \mathrm{mg} / \mathrm{dL}$ (22.5 to $47.2 ; P=.0023)$ and $118.1 \mathrm{mg} / \mathrm{dL}$ (59.4 to $176.7 ; P=.0016)$, respectively, in the collaborative care group. Significant changes in systolic and diastolic blood pressures and LDL cholesterol between groups was not observed.

Therapeutic diabetes outcomes were also evaluated and are depicted in Table 3. Rate of pneumococcal polysaccharide vaccine, 23 -valent (PPSV23) vaccinations increased by $19.5 \%$ in the collaborative care group versus $13.5 \%$ in usual care $(P=.026)$ while the usual care group had a larger increase in pneumococcal conjugate vaccine, 13-valent (PCV13) vaccination rates of $13.8 \%$ versus $7.8 \%$ in the collaborative group $(P=.0075)$. The usual care group had $74.0 \%$ of patients with $\mathrm{HbA1c}$ values less than $8 \%$ at follow-up, which was significantly higher than the collaborative care group at $64.7 \%(P=.0049)$. Aspirin and statin rates also increased for both groups yet were not found to be statistically significant.

Changes in the number of ED and hospital visits were assessed 12 months before and after the date of entry into the study. Both ED and hospital visits decreased significantly post-study entry in the collaborative care group. Emergency department visits displayed an overall increase of 23 visits in the usual care group versus a decrease of 22 visits in the collaborative care group $(P=.0162)$. Similarly, hospital visits increased by 37 visits in the usual care group and decreased by 18 visits in the collaborative care group $(P=.0225)$.

\section{Cost Analysis}

Using local cost estimates derived from Ascension Via Christi Hospitals Wichita average direct costs, the average hospital visit cost estimate was $\$ 10,962$ and the average emergency department visit cost 
Table 3. Changes in Diabetes Therapeutic Outcomes for Usual Care and Collaborative Care Groups

\begin{tabular}{|c|c|c|c|c|c|c|c|c|c|}
\hline & \multicolumn{4}{|c|}{ Usual Care } & \multicolumn{4}{|c|}{ Collaborative Care } & \multirow[b]{2}{*}{$\begin{array}{c}P \\
\text { Value }\end{array}$} \\
\hline & $\mathrm{n}$ & $\begin{array}{l}\text { Baseline, } \\
\text { No. (\%) }\end{array}$ & $\begin{array}{l}\text { Follow-up, } \\
\text { No. (\%) }\end{array}$ & $\begin{array}{l}\text { Change, } \\
\text { No. (\%) }\end{array}$ & $\mathrm{n}$ & $\begin{array}{l}\text { Baseline, } \\
\text { No. (\%) }\end{array}$ & $\begin{array}{l}\text { Follow-up, } \\
\text { No. (\%) }\end{array}$ & $\begin{array}{l}\text { Change, } \\
\text { No. (\%) }\end{array}$ & \\
\hline $\begin{array}{l}\text { PPSV23 } \\
\quad \text { vaccination }\end{array}$ & 385 & $18(4.7)$ & $70(18.2)$ & $52(13.5)$ & 385 & $47(12.2)$ & $122(31.7)$ & $75(19.5)$ & .0255 \\
\hline PCV13 vaccination & 385 & $66(17.1)$ & $119(30.9)$ & $53(13.8)$ & 385 & $121(31.4)$ & $151(39.2)$ & $30(7.8)$ & .0075 \\
\hline Aspirin* & 385 & $182(47.3)$ & $218(56.6)$ & $36(9.4)$ & 385 & $199(51.7)$ & $236(61.3)$ & $37(9.6)$ & .9021 \\
\hline Statin* & 385 & $177(46.0)$ & $259(67.3)$ & $82(21.3)$ & 385 & $196(50.9)$ & $291(75.6)$ & $95(24.7)$ & .2655 \\
\hline HbAlc of $<8 \%$ & 385 & - & $285(74.0)$ & & 385 & - & $249(64.7)$ & & .0049 \\
\hline
\end{tabular}

HbA1c, glycosylated hemoglobin; PCV13, pneumococcal conjugate vaccine (13-valent); PPSV23, pneumococcal polysaccharide vaccine (23-valent).

*Aspirin and statins included all drugs in the classes of salicylates and HMG-CoA reductase inhibitors, respectively.

estimate was \$669. The usual care group had an increase of $23 \mathrm{ED}$ visits and 37 hospital visits. The total cost of this increase in health care use in the usual care group was estimated to total an increase in cost of $\$ 420,981$ or $\$ 1093$ per patient. The collaborative care group had a decrease of $22 \mathrm{ED}$ visits and 18 hospital visits, displaying a substantial decrease in health care use with an estimated savings of $\$ 212,034$ or $\$ 551$ per patient. The total savings and cost avoidance for physician-pharmacist collaboration (collaborative care cost reduction added to usual care cost increase) was $\$ 633,015$ or $\$ 1644$ per patient for the 12-month follow-up period. The cost savings attributed to diabetes management financially justifies the salaries and benefits for our 2 clinical pharmacists. However, our pharmacists also provide a wide variety of other clinical services in addition to diabetes, making it difficult to estimate the cost of the diabetes management portion of our clinical services. It should be noted the cost of a collaborative diabetes management program can vary based on several factors including geographic location of the program, patient population, and other responsibilities of the clinical pharmacist.

\section{Subgroup Analyses}

We conducted a post-hoc subgroup analysis to explore the potential influence of varying baseline HbA1c values between the usual care and collaborative care groups. As noted in Table 1, the usual care group displayed a considerably lower average $\mathrm{HbA1c}$ at baseline as compared with the collaborative care group. This analysis was conducted only for the primary outcome of change in $\mathrm{HbAlc}$ from baseline to follow-up and was prepared by excluding previously matched cohort patients based on baseline level of control. Three groups were evaluated to analyze the potential influence of lower baseline HbAlc values on mean change in HbAlc at followup. Groups were evaluated with the exclusion of patients with a baseline HbA1c: 1) less than 7\%, 2) less than $8 \%$, and 3 ) less than $9 \%$. Mean HbA1c change from baseline to follow-up between the groups was then assessed (Table 4).

With each of the 3 exclusion groups in the posthoc analysis, the collaborative care group still showed a statistically significant decrease in HbA1c compared with the usual care group $(P<.0001)$.

\section{Discussion}

Our findings demonstrate that pharmacists providing disease state management in collaboration with physicians in a primary care setting is associated with improved diabetes outcomes and reduces health care costs. The reduction in HbA1c in the collaborative care group is both statistically and clinically significant when compared with the usual care group. As previous research from Stratton et $\mathrm{al}^{26}$ has demonstrated, a $1 \%$ decrease in $\mathrm{HbA1}$ c reduces the risk for myocardial infarction by $14 \%$, death related to diabetes by $21 \%$, and microvascular complications by $37 \%$. When extrapolating results from Stratton et $\mathrm{al}^{26}$ to the collaborative care population, the reduction in $\mathrm{HbAlc}$ of $1.75 \%$ potentially represents a reduction in risk of myocardial infarction, death related to diabetes, and microvascular complications of $24 \%, 36 \%$, and $64 \%$, respectively.

On data analysis, it was noted that the baseline $\mathrm{HbA1c}$ values in the usual care group were markedly 
Table 4. Subgroup Analysis with Exclusion of Varying Levels of Baseline HbA1c Control

\begin{tabular}{|c|c|c|c|c|c|}
\hline & $\mathrm{n}$ & Baseline, Mean \pm SD & Follow-up, Mean \pm SD & Change, Mean (95\% CI) & $P$ Value \\
\hline \multicolumn{6}{|l|}{ Baseline HbA1c $\geq 7 \%$} \\
\hline Usual care, HbA1c, \% & 222 & $8.50 \pm 1.53$ & $8.03 \pm 1.56$ & $0.47(0.28$ to 0.66$)$ & \multirow[t]{2}{*}{$<.0001$} \\
\hline Collaborative care, HbA1c, \% & 355 & $9.68 \pm 2.00$ & $7.79 \pm 1.60$ & $1.89(1.65$ to 2.13$)$ & \\
\hline \multicolumn{6}{|l|}{ Baseline HbA1c $\geq 8 \%$} \\
\hline Usual care, HbA1c, \% & 119 & $9.47 \pm 1.52$ & $8.56 \pm 1.72$ & $0.90(0.62$ to 1.18$)$ & \multirow[t]{2}{*}{$<.0001$} \\
\hline Collaborative care, HbA1c, \% & 284 & $10.24 \pm 1.84$ & $7.96 \pm 1.60$ & $2.29(2.02$ to 2.55$)$ & \\
\hline \multicolumn{6}{|l|}{ Baseline $\mathrm{HbA1c} \geq 9 \%$} \\
\hline Usual care, HbA1c, \% & 63 & $10.42 \pm 1.54$ & $9.14 \pm 1.95$ & $1.28(0.82$ to 1.73$)$ & \multirow[t]{2}{*}{$<.0001$} \\
\hline Collaborative care, HbA1c, \% & 204 & $10.95 \pm 1.70$ & $8.11 \pm 1.71$ & $2.84(2.51$ to 3.17$)$ & \\
\hline
\end{tabular}

HbA1c, glycosylated hemoglobin; SD, standard deviation; CI, confidence interval.

All data represented includes subjects originally matched for primary analysis with subjects excluded based on baseline HbA1c values. Subjects included in the subgroup with baseline HbA1c $\geq 9 \%$ are included in all three subgroup analyses based on meeting baseline HbA1c for each group. Subjects with baseline HbA1c of $\geq 8 \%$ are included in subgroup analyses with baseline $\mathrm{HbA1c}$ of $\geq 8 \%$ and $\geq 7 \%$.

lower than that of the collaborative care group and were lower than anticipated based on initial sample data. Higher baseline HbA1c values in the collaborative care group was not entirely unforeseen, as a large portion of the patient population referred to the clinical pharmacists often have significantly elevated HbA1c or are unable to obtain and maintain glycemic control for varying reasons. This observation also supports the significantly higher number of baseline ED and hospital visits seen in the collaborative care group as well as the greater number of patients with HbA1c values of less than $8 \%$ at follow-up in the usual care group. These findings guided the decision to perform a post-hoc subgroup analyses, using performance measures set forth by the National Committee for Quality Assurance as a guideline for HbAlc control. ${ }^{27}$ These analyses were performed to reduce potential bias associated with the lower baseline HbA1c values in the usual care group, as the mean baseline HbA1c was only slightly above the standard American Diabetes Association (ADA) HbAlc goal of less than $7 \% .{ }^{28}$ With the exclusion of varying levels of glycemic control, the collaborative care group displayed a significant reduction in HbAlc compared with the usual care group, further supporting the addition of a clinical pharmacist to primary care to improve diabetes outcomes.

While the change in pneumococcal vaccination rates for PPSV23 vaccinations between groups favored the collaborative care group, PCV13 vaccination rates displayed favor for the usual care group. Having a clinical pharmacist on the care team provides additional opportunities for review of vaccination status to ensure patients receive appropriate and timely vaccinations. Appropriate use of pneumococcal vaccinations has continued to be a source of confusion for many health care workers due to multiple recent changes in guidelines and has been a subject of education within our primary care clinics. It is important to note, however, that this study did not address the appropriateness of pneumococcal vaccinations.

Both ED and hospital visits decreased significantly post-study entry in the collaborative care group, rendering substantial cost savings and cost avoidance totaling $\$ 633,015$ for a 12 -month period. The addition of pharmacists in the primary care setting improves patient outcomes and reduces costs associated with health care use.

Health care in the United States has been driven by a fee-for-service payment model that encourages high volume productivity, provides little incentive for improving health outcomes, limits efficiency for primary care, and inherently drives up health care costs with a flawed payment system. ${ }^{29,30}$ With its many inadequacies, the fee-for-service payment model also makes it difficult to financially justify the addition of a clinical pharmacist to the primary care team. As health care transitions away from feefor-service and toward alternative payment models, ${ }^{29,30}$ team-based care has become more accepted to help improve quality, with pharmacists practicing as an essential member of the team. ${ }^{4,15}$ The significant cost savings associated with the reduction in all-cause ED visits and hospitalizations as well as 
the improvement in diabetes outcomes found in this study could be utilized to justify the addition of a pharmacist to a primary care clinic and support expansion of existing clinical pharmacist services. The 6 clinics included in the collaborative care group were served by 2 full-time clinical pharmacists. The estimated cost savings of $\$ 633,015$ for a 12 month period not only financially supports the salary and benefits for the current clinical pharmacists but provides further justification for additional pharmacists to expand clinical pharmacy services to the remaining primary care clinics within the health system. Our study supports the inclusion of a pharmacist in the team-based care model to improve diabetes quality metrics including $\mathrm{HbA1c}$ reduction and improved vaccination rates.

One of the strengths of this study is that patients in the collaborative care group were matched to those in the usual care group, thereby simulating comparable patients that would potentially be referred to a clinical pharmacist if those services were to have been available in the usual care group. This study, like many, is not without limitations. In addition to other limitations, our study did not address the changes in medication adherence and improved medication access that can occur with pharmacist involvement in the care team. Our clinical pharmacists use current guideline-directed medication therapy that includes many different oral and injectable medications to help patients reach glycemic goals; however, concomitant diabetes medications other than insulin therapy at study entry were not addressed. Additional confounding factors affecting $\mathrm{HbAlc}$ were not evaluated. We also did not evaluate total clinic visits to determine whether more frequent visits with a pharmacist would potentially affect the degree of $\mathrm{HbAlc}$ reduction, or whether the interaction with a new care team member affected the patient's motivation to improve glycemic control. Although this study did not exclude patients with an HbAlc of less than 7\%, which by ADA standards is considered controlled, the post-hoc subgroup analysis confirmed that excluding these patients from analysis did not impact the difference in mean $\mathrm{HbAlc}$ between groups, with the collaborative care group showing a considerable reduction in HbA1c compared with usual care, regardless of level of baseline $\mathrm{HbA1}$ c control.

\section{Conclusions}

The addition of clinical pharmacists in the primary care setting is associated with improved patient outcomes for diabetes and substantially reduced costs due to decreased health care use. The primary outcome showed a significant reduction in $\mathrm{HbA1c}$ in the collaborative care group compared with usual care. Post-hoc subgroup analysis revealed that significant $\mathrm{HbAlc}$ reductions were seen in the collaborative care group after exclusion of subjects with varying degrees of initial HbA1c control. Our findings support expanding ambulatory care pharmacy services to improve patient outcomes and reduce health care costs. Further studies are needed to explore additional confounding factors affecting changes in $\mathrm{HbAlc}$.

The authors would like to acknowledge the considerable assistance provided by Kenneth Utz, PharmD, BCOP for data acquisition; Hayrettin Okut, $\mathrm{PhD}$ for statistical analysis of the study data; and Tara Katz, MD for editorial assistance.

To see this article online, please go to: bttp://jabfm.org/content/ 33/5/745.full.

\section{References}

1. Hazen ACM, de Bont AA, Boelman L, et al. The degree of integration of non-dispensing pharmacists in primary care practice and the impact on health outcomes: A systematic review. Res Social Adm Pharm 2018;14:228-40.

2. Chua SS, Kok LC, Yusof FAM, et al. Pharmaceutical care issues identified by pharmacists in patients with diabetes, hypertension, or hyperlipideamia in primary care settings. BMC Health Serv Res 2012;12:388.

3. Jun JK. The role of pharmacy through collaborative practice in an ambulatory care clinic. Am J Lifestyle Med 2019;13:275-81.

4. Chisholm-Burns MA, Kim Lee J, Spivey CA, et al. US pharmacists' effect as team members on patient care: systematic review and meta-analysis. Med Care 2010;48:923-33.

5. Giberson S, Yoder S, Lee MP. Improving patient and health system outcomes through advanced pharmacy practice. A Report to the U.S. Surgeon General. Office of the Chief Pharmacist. Washington, DC: U. S. Public Health Service. 2011.

6. Brunisholz KD, Olson J, Anderson JW, et al. "Pharming out" support: a promising approach to integrating clinical pharmacists into established primary care medical home practices. J Int Med Res 2018;46:234-48.

7. Dixon DL, Sisson EM, Parod ED, et al. Pharmacist-physician collaborative care model and time to goal blood pressure in the uninsured population. J Clin Hypertens 2018;20:88-95.

8. Boykin A, Wright D, Stevens L, et al. Interprofessional care collaboration for patients with heart failure. Am J Health Syst Pharm 2018;75:e45-e49. 
9. Nagelkerk J, Thompson ME, Bouthillier M, et al. Improving outcomes in adults with diabetes through an interprofessional collaborative practice program. J Interprof Care 2018;32:4-13.

10. Schwartz EJ, Turgeon J, Patel J, et al. Implementation of a standardized medication therapy management plus approach within primary care. J Am Board Fam Med 2017;30:701-14.

11. Cox N, Tak CR, Cochella SE, et al. Impact of pharmacist previsit input to provider on chronic opioid prescribing safety.J Am Board Fam Med 2018;31:105-12.

12. Jones LK, Greskovic G, Grassi DM, et al. Medication therapy disease management: Geisinger's approach to population health management. Am J Health-Syst Pharm 2017;74:1422-35.

13. Gums TH, Carter BL, Milavetz G, et al. Physicianpharmacist collaborative management of asthma in primary care. Pharmacotherapy 2014;34:1033-42.

14. Centers for Disease Control and Prevention. Advancing team-based care through collaborative practice agreements: a resource and implementation guide for adding pharmacists to the care team. Atlanta, GA: Centers for Disease Control and Prevention, US Department of Health and Human Services; 2017.

15. Weaver K, Brookhart A, Kent J, et al. The team approach to health care. J Am Pharm Assoc 2016;56:616-9.

16. Pontefract BA, King BS, Gothard DM, et al. Impact of pharmacist-led diabetes management in primary care clinics. Innov Pharm 2018;9:17-8.

17. Matzke GR, Moczygemba LR, Williams KJ, et al. Impact of a pharmacist-physician collaborative care model on patient outcomes and health services utilization. Am J Health Syst Pharm 2018;75:1039-47.

18. Wubben DP, Vivian EM. Effects of pharmacist outpatient interventions on adults with diabetes mellitus: a systematic review. Pharmacotherapy 2008;28:421-36.

19. Ip EJ, Shah BM, Yu J, et al. Enhancing diabetes care by adding a pharmacist to the primary care team. Am J Health Syst Pharm 2013;877-86.
20. Hirsch JD, Steers N, Adler DS, et al. Primary carebased, pharmacist-physician collaborative medicationtherapy management of hypertension: a randomized, pragmatic trial. Clin Ther 2014;36:1244-54.

21. Funk KA, Pestka DL, Roth McClurg MT, et al. Primary care providers believe that comprehensive medication management improves their work-life. J Am Board Fam Med 2019;32:462-73.

22. Kulchaitanaroaj P, Brooks JM, Chaiyakunapruk N, et al. Cost-utility analysis of physician-pharmacist collaborative intervention for treating hypertension compared with usual care. J Hypertens 2017;35:178-87.

23. Sease JM, Franklin MA, Gerrald KR. Pharmacist management of patients with diabetes mellitus enrolled in a rural free clinic. Am J Health-Syst Pharm 2013;70:43-7.

24. Schultz JL, Horner KE, McDanel DL, et al. Comparing clinical outcomes of a pharmacist-managed diabetes clinic to usual physician-based care. J Pharm Pract 2018;31:268-71.

25. Santschi V, Chiolero A, Paradis G, et al. Pharmacist interventions to improve cardiovascular disease risk factors in diabetes. Diabetes Care 2012;35:2706-17.

26. Stratton IM, Adler AI, Neil HA, et al. Association of glycaemia with macrovascular and microvascular complications of type 2 diabetes (UKPDS 35): prospective observational study. BMJ 2000;321:405-12.

27. National Committee for Quality Assurance. Comprehensive diabetes care (CDC 2019). www. ncqa.org/hedis/measures/comprehensive-diabetescare. Accessed December 23, 2019.

28. American Diabetes Association. Standards of medical care in diabetes. Diabetes Care 2019;42:S61S70.

29. Schroeder SA, Frist W. Phasing out fee-for-service payment. N Engl J Med 2013;368:2029-32.

30. Bazemore A, Phillips RL, Glazier R, Tepper J. Advancing primary care through alternative payment models: lessons from the United States and Canada. J Am Board Fam Med 2018;31:322-7. 